Session 2632

\title{
Introduction to Electrical Engineering: A Freshman/Sophomore- Level Course Covering the Breadth of Electrical Engineering
}

\author{
Alan V. Sahakian \\ Northwestern University
}

\begin{abstract}
We describe our new introductory course in Electrical Engineering, which covers the breadth of the field, rather than in in-depth treatment of circuits. A single unifying example, the CD player, is used to introduce the fundamental areas and their applications. Digital signal processing, coding and communications, control systems, optics, solid state, and electronics are all seen at work in a familiar electronic device. Seven laboratory experiments are included to reinforce the lectures. Our experience after two offerings has been positive, with both EE and non-EE engineering students enrolled.
\end{abstract}

\section{Introduction}

The traditional entry-level course to an Electrical Engineering (EE) curriculum is circuits. Here students learn important concepts and tools which serve them well in later courses and their careers, but they are not exposed to the many exciting areas of EE which are reshaping the world. Some may not see the relevance of this material and begin to lose their enthusiasm.

We have introduced a new EE core curriculum starting with a fundamentals course, ECE 202, which introduces the breadth and applications of $\mathrm{EE}^{1}$. We chose a familiar unifying example, the Compact Disc (CD) player, as the vehicle in ECE 202 to convey the importance and applications of some of the principles in the fundamental areas of EE. We introduce circuits, signals, communications and coding, control systems, electromagnetics and optics, devices and materials and show the relevance of each of these by referring in detail to the CD example. Our goal is for students to gain an understanding of these fields and their applications and to learn some of the most important basic concepts and quantitative tools in each. They also learn the principles which lead to an understanding of the present limits and EE challenges for the future. The course includes seven 3-hour laboratory experiments. It is followed by more traditional core EE courses which take the students further in each fundamental area.

II. Organization

Proceedings of the 2001 American Society for Engineering Education Annual Conference \& Exposition Copyright (O) 2001, American Society for Engineering Education 
This is a ten-week course with four lectures per week. The first week describes the operation of a $\mathrm{CD}$ player at a block-diagram level. The major elements are explained in terms of the functions they perform, and the challenges faced in their design. For example, the challenge of tracking and focus, and the need for feedback control.

The second week (re-)introduces some the basic circuit material which students will have seen in Physics. Voltage, current, resistance, power and energy are defined. The capacitor and inductor are introduced as circuit elements and described by their current-voltage differential equations. Simple first-order circuits are analyzed in the time domain. Reduction of parallel and series combinations is described.

This is followed in the third and fourth weeks with a discussion of signals and systems concepts. The idea of a sampled signal is introduced and concepts of spectral representation and aliasing are described. Quantization and signal-to-noise ratio are introduced and examples of digital music sampled at different rates and with different resolution are played to hear the effects. The properties of linearity and time invariance are introduced. Simple difference equations implementing digital filters are examined and demonstrated, although the $\mathrm{z}$ transform is not described.

Communications is introduced in the fifth week. Analog modulation schemes and their spectral characteristics are described. These include AM, FM, PM and SSB, and the CD recording scheme is seen to be an example of FM. Digital modulation techniques such as ASK and DPSK are introduced. Physical limits, such as Shannon's capacity theorem and the effects of signal-to-noise ratio are discussed. At this point, some simple problems involving performance in digital wireless communication are worked. An example is the effect of varying distance between a base station and a handheld unit on the capacity.

Along with digital communications, error-control coding schemes are introduced and the Hamming code is described in detail. We found that the actual error-control scheme used in the $\mathrm{CD}$ standard is too complex to use as a teaching example, but the Hamming code shows the principle.

In the sixth week feedback control systems are introduced. The source of the feedback signals (the differential optical sensors and associated optical components) in the CD player are described. Proportional, and proportional/integrating control are introduced. Instability and error are shown in example simulations, but the requirements for stability are not discussed.

In the seventh and eighth weeks, optical components, including lasers and photodetectors, are described. Basic optical properties are introduced, including Snell's law, total internal reflection, attenuation and diffraction. Aside from the CD player, fiber optics and free-space optical communication are used as examples. The capacity of CD players, and some of the newer standards such as CD-RW and DVD are described. 
The ninth week introduces electronic devices and their applications. Transistors are defined in terms of their current-voltage relationships (not their physical principles), and the ideal operational amplifier and simple circuits using it are shown.

Finally, the tenth week of the course introduces materials and the fabrication of devices. Processes such as MOCVD and MBE are described, and the steps used to fabricate lasers, detectors, and semiconductors in general are shown.

III. Labs

The seven laboratory experiments are scheduled to just follow the relevant lecture material. These are:

The inner workings of a CD player

Elementary circuits and instrumentation

Digital signal processing

Communications

Control systems

Optics

Lasers and photodetectors

For example, in the first experiment, students disassemble a cd player and examine its operation. The effects of defects on the disc surface are explored by using a dry erase marker. Students are asked to identify and describe the various components.

IV. Results

We have offered this course twice, as a pilot during the winter quarter of 2000 and as a full-scale course during the Fall of 2000. In the latter there were 65 students, mostly ECE but also BME, $\mathrm{ME}$ and even one adventurous Psychology student. Students were evaluated by one midterm and a final. The outcome was positive, with no student performing below passing. The other component of our evaluation is tracking performance in the next course, circuits, which is being offered during the current winter quarter.

Student evaluations of the course were largely but not entirely positive. The majority felt that the example was interesting and that compared to taking circuits as the first course, much less dry. The negative comments were, for the most part, focused on the breadth of the course. That is that there were too many topics covered in ten weeks.

\section{Conclusions}

We have described a first course in the EE curriculum which introduces students to some of the 
applications of the advanced course material which they will be learning. Our experience with the course has been positive, but we plan to narrow the scope a bit to keep it a reasonable ten-week offering.

Bibliography

1. URL: http://www.ece.nwu.edu/ sahakian/202/

\begin{abstract}
ALAN V. SAHAKIAN
Alan V. Sahakian received his BS in Applied Sciences and in Physics from the University of Wisconsin - Parkside, and his MSEE in 1979 and Ph.D. in Electrical Engineering in 1984 from the University of Wisconsin - Madison. Since then he has been on the faculty of Northwestern University, where is he is currently Professor of Electrical and Computer Engineering, and of Biomedical Engineering, and the Director of the EE Undergraduate Program, and holds the Charles Deering McCormick Professorship in Teaching Excellence. He has also worked as a Senior Electrical Engineer at Medtronic, Inc.
\end{abstract}

